\title{
Forecasting of Electricity Demand by Hybrid ANN-PSO under Shadow of the COVID-19 Pandemic
}

\author{
Mohamed Rahmoune*, Saliha Chettih \\ Department of Electrical Engineering, University of Amar Telidji Laghouat, Laghouat 03000, Algeria
}

Corresponding Author Email: rahmounemed@yahoo.fr

https://doi.org/10.18280/ejee.230602

Received: 6 October 2021

Accepted: 2 December 2021

\section{Keywords:}

particle swarm optimization, artificial neural network, short term load forecasting, COVID19

\begin{abstract}
Here in the research paper, we did not use smart methods to predict the future but rather to show the impact of the pandemic, we used the hybrid method using the PSO-ANN algorithm to demonstrate the impact of COVID-19 on electricity consumption and to demonstrate that we used two basic steps. The first step is to demonstrate that the hybrid method is effective for prediction. We showed that the prediction for 2019 was good, and that was before the onset of COVID-19. As for the second step, we applied the same hybrid algorithm after the emergence of COVID-19, i.e. for 2020, to note the difference between the prediction and the current pregnancy, which represents the impact of this epidemic, and this prediction in the short term. A short-term role in the operation of a power system in terms of achieving an economical electrical output and avoiding losses or outages. We've collected four consecutive years of data that is downloaded every quarter-hour of the day. Electricity consumption in Algeria is used as an input to the PSO-ANN learning algorithm. The results of the PSO-ANN pregnancy prediction algorithm have better accuracy than the ANN prediction. In the future but with the emergence of a pandemic that has had a clear difference and represents economic losses in the field of electricity, the epidemic should be viewed as a short-term variable to reduce the level of energy loss and generation cost.
\end{abstract}

\section{INTRODUCTION}

Since December 2019, an outbreak of pneumonia from unknown causes has been appeared for the first time in Wuhan, Hubei Province of China [1]. After the apparition of these symptoms, the World Health Organization (WHO) has been signaled it as a new pandemic known as COVID-19 (SARSCoV-2). On 12, February 2020, there are 43, 103 confirmed cases where more than $99 \%$ are from China. However, several governments around the world have been imposed severe restrictions to face this new situation $[2,3]$.

In February 2020, Algeria government has been declared the first case of this pandemic in Blida city. Then, as a first solution, they tried to impose strict restrictions in order to limit the spread of this pandemic. Nevertheless, this latest has been spread more day after day throughout the country. On $22^{\text {nd }}$, March 2020, the Algerian government has been imposed a second plan based on the lockdown of its borders (sea, air and land) excepting the very important ones. Through this new situation, almost airports (both national and international trips), roads (both principal and secondary), and seaport were practically empty, universities, colleges, public cafe, restaurants, shops, and industrial activities were mostly closed. As this severe lockdown rules, Algerian company of power system named as SONELGAZ has been recorded a drop in energy consumption.

From the above presented situation, there are several technical reports and research papers that studied the impacts of COVID-19 in the load demand.

The diminution of load consumption due to decreasing demand from both services and industrial sectors when the lockdown applied was affirmed by the International Energy Agency (IEA) [4]. In the other hand, the Earth Institute (EI) of Columbia university was demonstrated that the load consumption has been increased in the domestic household sector $[3,5]$.

Aruga et al. [3], have presented a practical study of load demand decreasing in Indian power system by applying the Autoregressive Distributed Lag (ARDL) model in order to estimate the difference between the real and estimated consumption.

Chhetri [6] has studied and presented the effect of COVID19 on household load consumption in staff residence at the College of Science and Technology (CST), Phuentsholing, Bhutan by comparing their obtained results with the past two years.

Forecasting is a prerequisite of electrical power system operations to achieve the goal of planning and optimal operation of power systems if the forecast period ranges from a few hours to weeks called short-term load forecasting and is a necessary condition for controlling and scheduling power system operations to maintain load flow and emergency analysis.

Forecasting refers to the use of history, through analysis and research, to explore the evolution and changes of things, and to make high-level assessments and judgments about the future development of things. Predicting the load of a power system refers to the research or use of a set of mathematical methods to systematically process past and future loads under conditions of taking into account some important system 
operating characteristics, expansion decisions, natural conditions, social influences and meeting certain accuracy requirements. Load value at a specified time in the future. Improve the level of load forecasting technology that helps manage planned power, logically arrange network operation methods and unit maintenance plans, reduce power generation costs, formulate power building plans, and improve the economic and social benefits of system power. For electricity sales companies, it is useful for electricity sales companies to decide on medium and long-term contract signing strategies, transaction strategies, and user economic accounts. Therefore, load forecasting has become an important part of achieving real-time sales transformation and power system management modernization at the lowest cost. Short-term load forecasting plays a major role in fully powering up energy sales in the spot market. It includes basic power generation plans, system security analysis, and next day market and intraday so that the power company can reduce the deviation between its reported power and real energy consumption.

For this purpose, an attempt has been made in this paper to studied and examined the effect of COVID-19 during the lockdown period on the total load demand of the all-Algerian power system by introducing the STLF technique based on ANN-PSO model. We have mainly compared the obtained results with the same periods of the past year. For more realistic study, the same ANN-PSO model has been taken into consideration.

\section{PROBLEM FORMULATION}

\subsection{Particle swarm optimization}

The optimization algorithms of particle swarm were introduced in 1995 by Mekhamer et al. [7]. The idea of the particle swarm algorithm arose from studying the predation behavior of bird/fish and bee communities. It mimics the behavior of a flock of birds that fly in search of food. The collective cooperation of birds enables the group to achieve the optimal goal. It is an optimization method based on swarm intelligence. Genetic Algorithm "Mutation". He finds the best value by following the best value he currently has. Compared with other modern optimization methods, the obvious advantages of particle swarm optimization are fewer parameters that need to be modified, simple and easy to implement, and fast convergence. It is a new evolutionary algorithm developed in recent years. It is an iterative optimization tool. In the process of implementing the algorithm, there is no cross-mutation process, and the best particle is found in the solution space. The advantages of PSO are simple operation, easy implementation, simple algorithm parameters, and no need for complex modification.

Particle swarm optimization (PSO) algorithm is a stochastic optimization technique based on swarm, which was proposed by Esmin et al. [8]. PSO algorithm simulates animal's social behavior, including insects, herds, birds and fishes. These swarms conform a cooperative way to find food, and each member in the swarms keeps changing the search pattern according to the learning experiences of its own and other members [9].

At each iteration of each particle considered himself as the best position (Pbest). Global optimal value (Gbest) could be identified after a search of the Pbest. Velocity and position update equation of the particle that has been optimized by adding inertial $(\omega)$ It is as follows [10]:

$$
\begin{gathered}
\mathrm{v}(\mathrm{t}+1)=\mathrm{w}(\mathrm{t}) * \mathrm{v}(\mathrm{t})+\mathrm{c} 1 * \operatorname{rand} 1(\mathrm{t}) *(\operatorname{pbest}(\mathrm{t})+\mathrm{x}(\mathrm{t})) \\
+\mathrm{c} 2 * \operatorname{rand} 2(\mathrm{t}) *(\operatorname{Gbest}(\mathrm{t})-\mathrm{x}(\mathrm{t})) \\
\mathrm{x}(\mathrm{t}+1)=\mathrm{x}(\mathrm{t})+\mathrm{v}(\mathrm{t}+1)
\end{gathered}
$$

Variable to control the speed of the particle is called inertia $(\omega)$ parameters. These parameters can be said to have a good performance, if it has a range of 0.4 up to 0.9 . Variable parameters $\mathrm{c} 1$ and $\mathrm{c} 2$ are the cognitive and social, which can be calculated by the equation:

$$
\begin{aligned}
& \mathrm{c} 1(\mathrm{t})=\mathrm{c} 1 \max -\mathrm{c} 1 \min ((\text { Itermax- } \\
& \text { Iter }(\mathrm{t})) / \text { Ietrmax })+\mathrm{c} 1 \min \\
& \mathrm{C} 2(\mathrm{t})=\mathrm{c} 2 \max -\mathrm{c} 2 \min ((\text { Itermax- } \\
& \text { Iter }(\mathrm{t})) / \text { Ietrmax })+\mathrm{c} 2 \min
\end{aligned}
$$

Figure 1 shows the PSO algorithm, and notice that the optimization logic in it searches for minimums and all position vectors are assessed by the function and to clarify more, we likened to a swarm of fish.

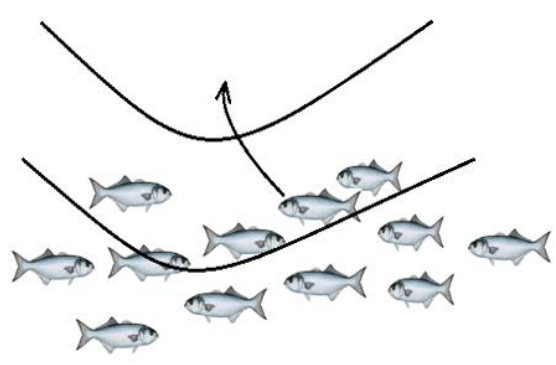

Figure 1. PSO Algorithm concept

\subsection{Backpropagation algorithm}

Biological neurons are cell bodies with dendrites, axons, and synapses. The dendrites can be thought of as input terminals, the electrical signals sent from other cells are transmitted; The axes can be considered the ends of the output, converting charges to other cells; Connecting neurons and a single neuron can be linked to thousands of neurons. There is a membrane potential in the cell body, and the current passing from outside causes the membrane to change and accumulate. When the transmembrane voltage rises above the threshold, the neuron is activated, generating an impulse, and passing it on to the next neuron. In order to more clearly understand the process of neuron signaling, compare a neuron to a reservoir of water. There are several water pipes (holes) attached to the bottom of the tank. The water pipe can not only drain the water in the tank (damper), but also inject water from the other tank (agitator). The thickness of the water tube is the change in the degree of impact of the water (weight). The change in the water level (membrane potential) of the tank by the water pipe is the change in the water level in the tank. When the water in the tank reaches a certain height, it can be drained through another tube (axon).

Artificial Neural Network (ANN) was introduced by Abdullah et al. [11]. This is the basic structure of a typical layered neural network. Layer L1 is the input layer, L2 is the hidden layer, and L3 is the hidden layer. Now we have a set of data $\mathrm{x} 1, \mathrm{x} 2, \mathrm{x} 3, \ldots, \mathrm{xn}$, and the result is also a set of data $\mathrm{y} 1$, $y 2, y 3, \ldots, y n$, now they are required to do some transformation in the hidden layer, so you can get the output expected after the data is poured into it. Including some variants. If your 
output is different from the original input, it's a very common artificial neural network, which is equivalent to letting the original data pass a mapping to get the output data we want, which is the topic we're going to talk about today.

Researchers only use a single-layer network architecture. BP algorithm was introduced by Leonard et al. [12]. In 1986, Rumelhart continued the BP algorithm for the hidden layer. This algorithm has a parameter called the learning rate for the control convergence algorithm for optimal local solution [13].

The BP training algorithm is divided into three stages, which are as follows.

Feed forward. We use predefined activation functions to calculate advanced input patterns from the input layer to the output layer.

It is the backpropagation. to calculate the error, we calculate the difference between the network output and the desired target. This error is transmitted directly back to the module in the output layer.

Weighing helps reduce errors.

Figure 2 shows the advanced stage structure and the posterior diffusion used throughout the study.

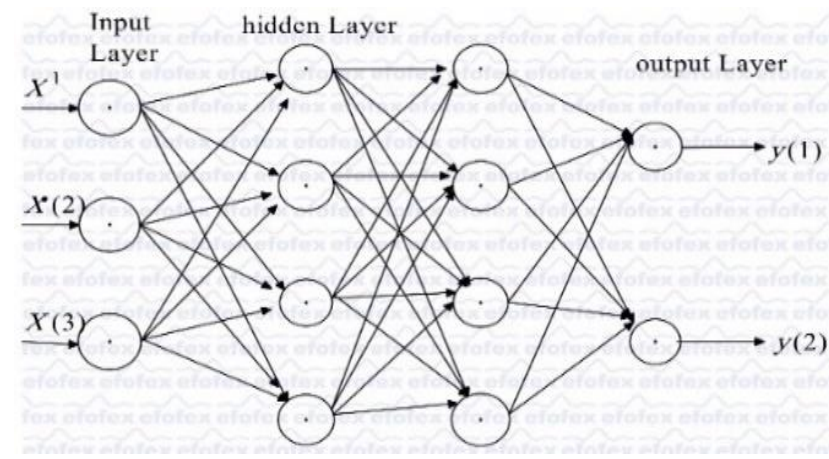

Figure 2. Backpropagation architecture

\subsection{Load forecasting methods}

Input data using the daily electricity every quarter of an hour load in 2017 until 2020 from the Algerian (Sonelgaz) Power Company.

Data are grouped, ANN algorithm used is the feedforward backpropagation algorithm (BP).

The calculation begins with the steps:

1-Group data and Normalization.

2-Initialization of the swarm particle.

3-Create Network Feedforward ANN.

4-Forecasting initial.

5-Evaluate fitness value (Gbest and Pbest).

6-Create Network of Backpropagation ANN.

7-Final Forecasting.

The calculation begins with the steps PSO algorithm is used to optimize the weights:

1-determine the initial position and velocity of particles.

2-number of particles.

3-the upper limit and lower limit of the search space and the number of iterations.

4-The position is the weight.

Weights of PSO are used as the optimal weights and biases in ANN. Learning rate parameter used 0.8. The maximum value of the epoch is 10000 .

\section{MODEL VERIFICATION EVALUATION}

The performances of the trained models, which have been employed in this study, were evaluated using the Root Mean Square Error (RMSE) and Mean Absolute Error (MAE). The mathematical expressions of RMSE and MAPE are as follows:

$$
\begin{aligned}
& \text { MAPE }=\frac{1}{n} \sum_{m=1}^{n} \frac{\left|\widehat{y_{t}}-y_{t}\right|}{y_{t}} * 100 \\
& \mathrm{RMSE}=\frac{1}{n} \sqrt{\sum_{m=1}^{n}\left(\left(\widehat{y}_{t}\right)-y\right)^{2}}
\end{aligned}
$$

In the formula above, $\mathrm{n}$ is the amount of total forecasting points with:

$\mathrm{n}$ is the amount of total forecasting points;

$\widehat{y_{t}}$ is the load forecasting;

$y_{t}$ is the load actual.

\section{PROBLEM SOLUTION}

Algeria has been considered as a developing country that having a considerable growth in electricity loads demand and production (Figure 3). This latest has been resulted due to several conditions such as:

The growing of both industry and business-based sectors.

The increasing of the Algerian population, etc.

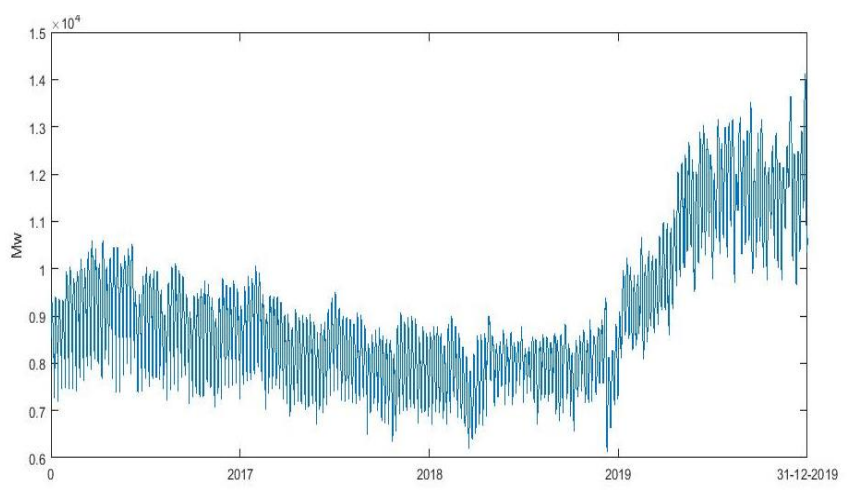

Figure 3. The Algerian data of quarter hour value every day (Jan 2017-Dec 2019)

The performance of this developed method for short-term load forecasting has been tested by using the MWh-actual load demand of the Electricity Generating Authority of the Algerian (OSE) the value of morning grandeur and the value of evening grandeur were loaded of day observations for electricity demand in Algeria: from January 2017 to December 2019.

In this study, we have tried to verify and check the impact of the COVID-19 pandemic in the load consumption of Algerian power system. To reach this goal, we have applied the ANN-PSO based method. For this reason, we have test and compare two main cases. In the first one, we have predicted the load by applying the STLF model during one day in February and April of 2019 in order to validate our ANN-PSO. Then, to make a fair comparison, we have applied the same ANN-PSO in the same days of the 2020 year to demonstrate the impact of COVID-19 in load consumption. After that, we have compared the obtained results in both cases before and after COVID-19 apparition with the real consumed load shown in figures. For the ANN model, we have used the feed forward back propagation and radial basis function to train the 
clustered data. In addition, we have used the real load data as an input and future consumed load as an output. In order to better validate the suggested model, we have separated the data into three subsets as follows:

1 -Training set $(60 \%)$ to create the model.

2-Validation set $(20 \%)$ to optimize the hyper-parameters and to avoid the over fitting.

3 -Testing set $(20 \%)$ to test the model.

All the simulations are performed by MATLAB software, on PC that having 64-bit operating system and Intel double core 2.16-GHz 2.16 - GHz processor.

Figures 4 (a-b-c) show the simulation results of load forecasting on day Randomly selected from the year a forecasting in contrast PSO-ANN and load actually at every quarter-hour. Input learning using electricity load, The ANN architecture has 10-5-1 layers and a learning rate of 0.8 .

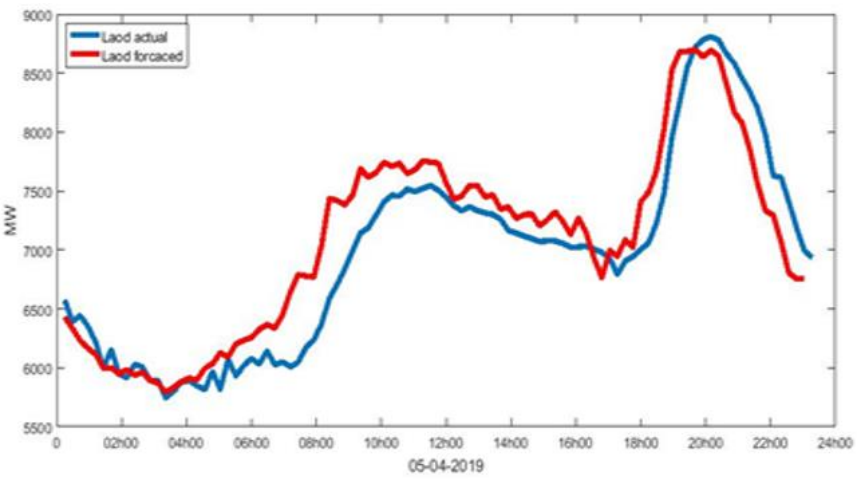

(a)

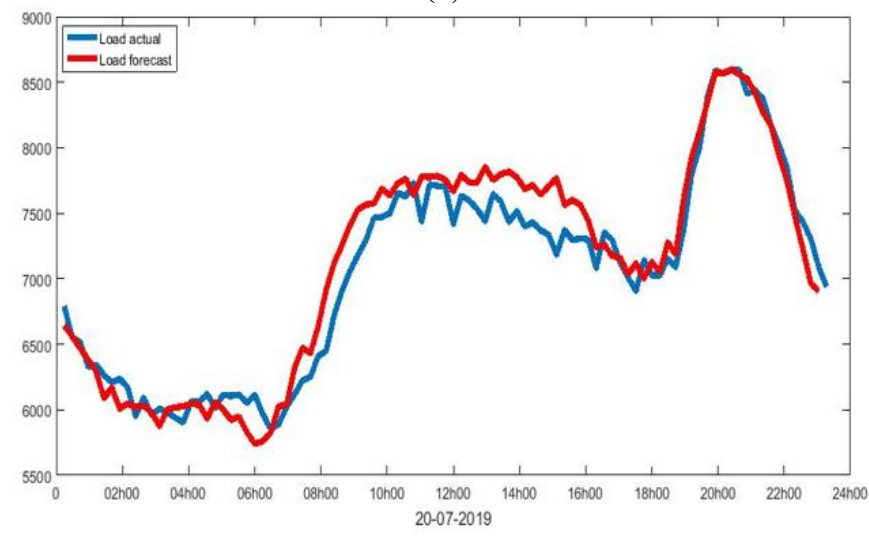

(b)

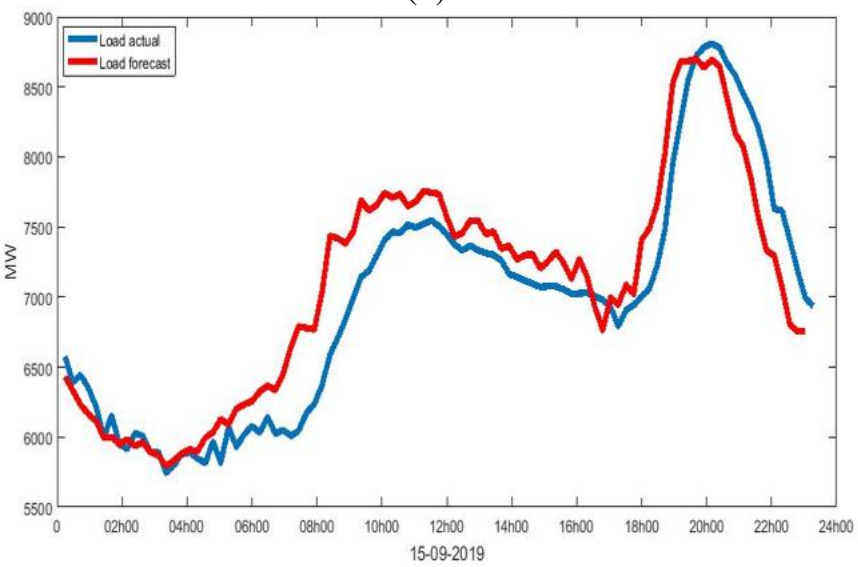

(c)

Figures 4. The simulation results of load forecasting on day Randomly selected from the year a forecasting in contrast PSO-ANN
The PSO-ANN day algorithm outperforms the forecasting algorithm in terms of load predicting accuracy. The absolute value of MAPE Obtained PSO-ANN is 2.01.

\subsection{Expectations with the emergence of COVID-19}

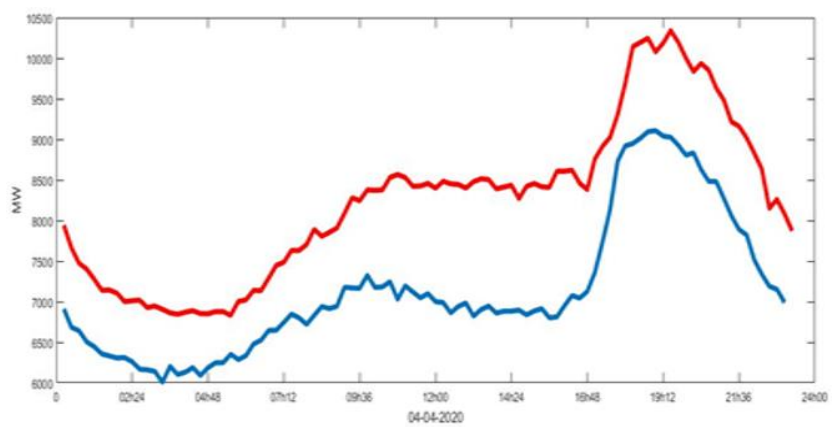

(a)

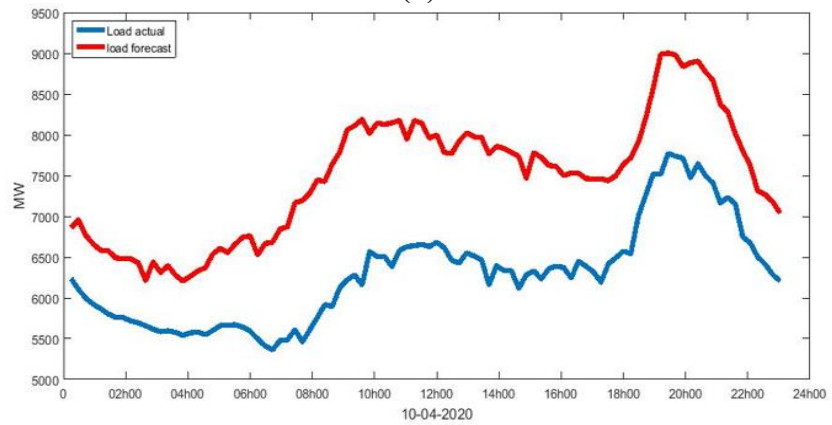

(b)

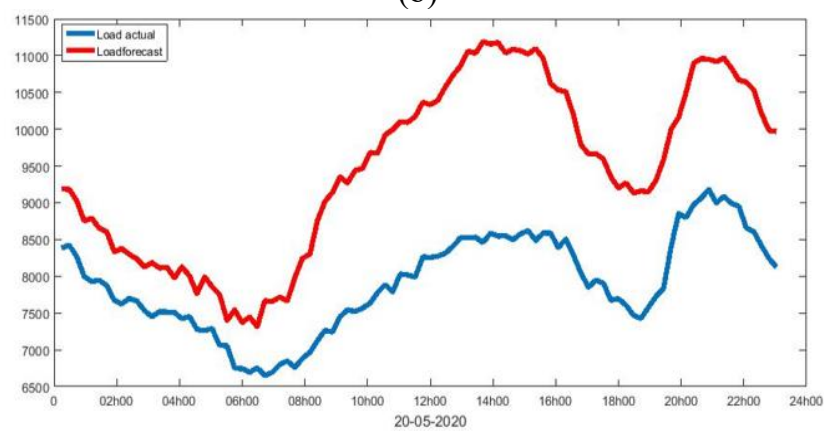

(c)

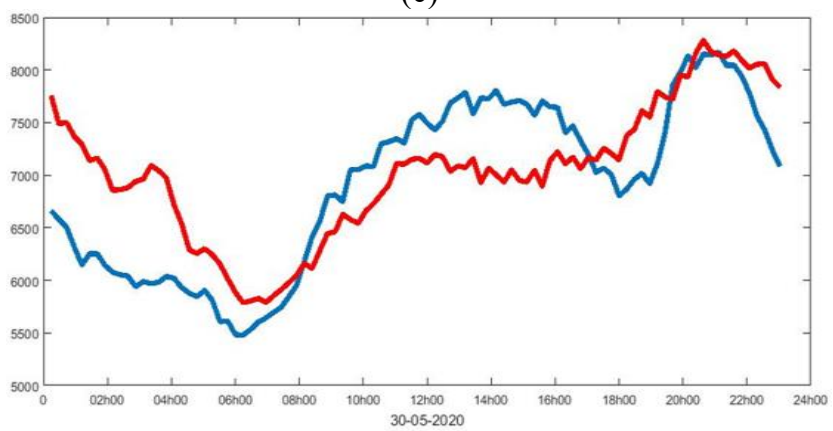

(d)

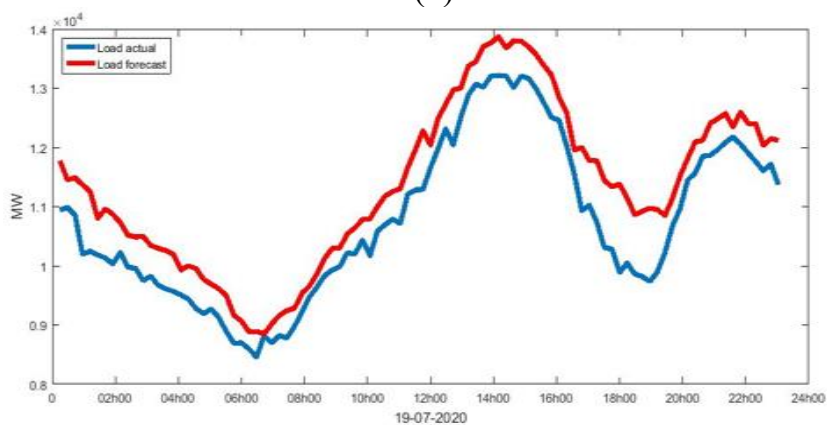

(e) 


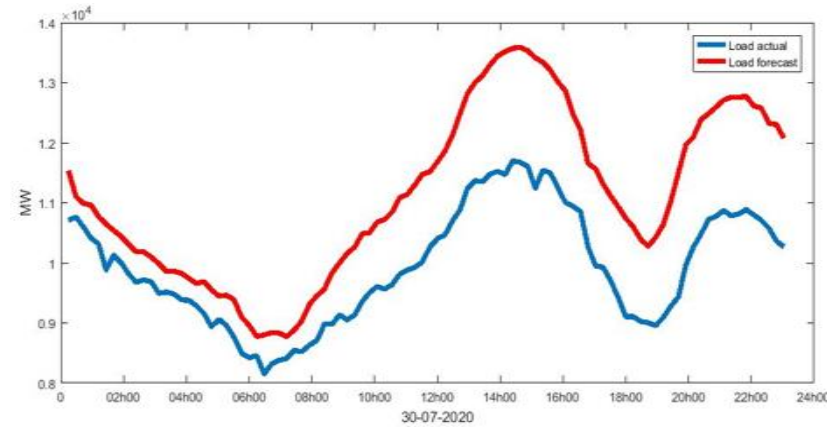

(f)

Figure 5. The simulation results of load forecasting on day Randomly selected from the year a forecasting in contrast PSO-ANN In the days of a pandemic

Figures 5 (a-b-c-e-f) show the simulation results of load forecasting on day Randomly selected from the year a comparison of the forecasting PSO-ANN and load actually at every quarter-hour Which is under the pandemic. Input learning using electricity load, while the ANN architecture using layers of 10-5-1 and learning rate 0.8. Load forecasting results show that the PSO-ANN day algorithm gives less accuracy than the forecasting that we interpreted as a pandemic effect.

The absolute value of MAPE Obtained PSO-ANN is 6.78.

The results are very interesting observed from the effects of load forecasting. The forecasting findings show a very substantial error of 6.78 percent, implying a loss of operational producing huge losses, particularly in energy and electricity generation costs.

Findings from simulations indicate COVID-19's impact on electrical energy consumption.

Inaccurate forecasting findings have a significant environmental impact, Algerian power generating is typically a thermal unit that uses gas and coal. with greater pollution projected as a result.

Therefore, any pandemic can be considered as a short-term impact factor on electricity consumption in the future.

From Table 1 (See Appendix), some conclusions can be drawn:

Obviously, month January models are more accurate than February, month January are more accurate than July, with a maximum MAPE error of $2.48 \%, 2.93 \%, 3.23 \%, 4.02 \%$, $3.78 \%, 10.13 \%$, and $7.38 \%$ respectively, the performance of the PSO-ANN is not good with a mean squared error value of $4.85 \%$. This indicates that the neural network predicts the output unsatisfactorily for the first four months of the year 2020 because the more widespread of a pandemic makes it even more difficult in terms of the uncertainty.

\section{CONCLUSION}

Since the COVID-19 has been propagated out worldwide, Algerian government had imposed a severe restriction based on stay-at-home by the end of March 2020. However, the energy consumption has been decreased significantly due to these regulations. Therefore, the energy consumption has been recovered when the relaxed rules were applied.

This article treats the problem and checks the impact of the lockdown procedure in Algerian power consumption during the apparition of COVID-19 pandemic by employing hybrid the ANN and PSO model. For this purpose, two cases have been tested and compared. In the first case, we have validated our proposed STLF approach by applying in the Algerian network during one day in February and April of 2019. After that, the same proposed ANN model has been used in the same days of the 2020 year in order to illustrate the impact of COVID-19 apparition, to illustrate the impact of a pandemic, we demonstrated the accuracy of the prediction of the use of neural networks before the pandemic for the year 2019, and then we applied the same network structure to demonstrate the effect of a pandemic on consumption for the year 2020, where we noticed a difference between prediction and consumption, from which we deduced the extent of the impact of a pandemic.

Finally, the obtained results clearly demonstrate the impact of the COVID-19 pandemic on the load consumption of Algerian power system due to the lockdown procedure of both industries and domestically sectors.

\section{REFERENCES}

[1] Zhang, Y.F., Ma, Z.F. (2020). Impact of the COVID-19 pandemic on mental health and quality of life among local residents in Liaoning Province, China: A crosssectional study. Environmental Research and Public Health, 17(7): 2381. https://doi.org/10.3390/ijerph17072381

[2] Jain, A.K., Duin, R.P.W., Mao, J. (2000). Statistical pattern recognition: A review. IEEE Transactions on Pattern Analysis and Machine Intelligence, 22(1): 4-37. https://doi.org/10.1109/34.824819

[3] Aruga, K., Islam, M., Jannat, A. (2020). Effects of COVID-19 on Indian energy consumption. Sustainability, $12(14)$ : 5616. https://doi.org/10.3390/su12145616

[4] COVID-19 Impact on Electricity. Available online: https://www.iea.org/reports/covid-19-impact-onelectricity, accessed on 16 June 2020

[5] Meinrenken, C.J., Modi, V., Mckeown, K.R., Culligan, P.J. (2020). New data suggest COVID-19 is shifting the burden of energy costs to households. Columbia University.

[6] Chhetri, R. (2020). Effects of COVID-19 pandemic on household energy consumption at college of science and technology. International Journal of Scientific Research and Engineering Development, 3(4): 1383-1387.

[7] Mekhamer, S.F., Soliman, S.A., Moustafa, M.A., ElHawary, M.E. (2003). Application of fuzzy logic for reactive-power compensation of radial distribution feeders. IEEE Transactions on Power Systems, 18(1): 206-213. https://doi.org/10.1109/MPER.2002.4311830

[8] Esmin, A.A., Coelho, R.A., Matwin, S. (2015). A review on particle swarm optimization algorithm and its variants to clustering high-dimensional data. Artificial Intelligence Review, 44(1): 23-45. https://doi.org/10.1007/s10462-013-9400-4

[9] Wang, D., Tan, D., Liu, L. (2018). Particle swarm optimization algorithm: An overview. Soft Computing, 22(2): 387-408. https://doi.org/10.1007/s00500-0162474-6

[10] McCulloch, W.S., Pitts, W. (1943). A logical calculus of the ideas immanent in nervous activity. Bulletin of Mathematical Biophysics 5: 115-133. https://doi.org/10.1007/BF02478259

[11] Abdullah, A.G., Suranegara, G.M., Hakim, D.L. (2014). Hybrid PSO-ANN application for improved accuracy of 
short-term load forecasting. WSEAS Transactions on Power Systems, 9(1): 446-451

[12] Leonard, J., Kramer, M.A. (1990). Improvement of the backpropagation algorithm for training neural networks. Computers \& Chemical Engineering, 14(3): 337-341. https://doi.org/10.1016/0098-1354(90)87070-6
[13] Zhang, J.R., Zhang, J., Lok, T.M., Lyu, M.R. (2007). A hybrid particle swarm optimization-back-propagation algorithm for feedforward neural network training. Applied Mathematics And Computation, 185(2): 10261037. https://doi.org/10.1016/j.amc.2006.07.025

\section{APPENDIX}

Table 1. Comparing MAPE of forecasting based on proposed ANN-PSO model for the months of January and February 2020 under shadow of the COVID-19 pandemic

\begin{tabular}{|c|c|c|c|c|c|c|c|}
\hline $\begin{array}{l}\text { Month of } \\
\text { January }\end{array}$ & $\begin{array}{c}\text { Daily actual } \\
\text { peak load }\end{array}$ & $\begin{array}{c}\text { Daily Load peak } \\
\text { forecast }\end{array}$ & MAPE \% & $\begin{array}{l}\text { Month of } \\
\text { February }\end{array}$ & $\begin{array}{c}\text { Daily actual } \\
\text { peak load }\end{array}$ & $\begin{array}{c}\text { Load } \\
\text { forecast }\end{array}$ & MAPE \% \\
\hline $01-01-2020$ & 9181 & 8970 & 2.30 & $01-02-2020$ & 9304 & 9343 & 0.42 \\
\hline 02-01-2020 & 9307 & 9294 & 0.14 & $02-02-2020$ & 9043 & 9202 & 1.75 \\
\hline $03-01-2020$ & 8861 & 8852 & 0.10 & $03-02-2020$ & 9111 & 9292 & 1.99 \\
\hline 04-01-2020 & 10025 & 9894 & 1.31 & $04-02-2020$ & 9445 & 9262 & 1.94 \\
\hline $05-01-2020$ & 10043 & 9974 & 0.69 & $05-02-2020$ & 9276 & 9462 & 2.01 \\
\hline $06-01-2020$ & 10124 & 10212 & 0.87 & $06-02-2020$ & 8974 & 9358 & 4.28 \\
\hline $07-01-2020$ & 10130 & 10130 & 0.00 & $07-02-2020$ & 9414 & 9656 & 2.57 \\
\hline $08-01-2020$ & 9450 & 10151 & 7.42 & $08-02-2020$ & 9443 & 9478 & 0.37 \\
\hline $09-01-2020$ & 9666 & 10191 & 5.43 & $09-02-2020$ & 9443 & 9051 & 4.15 \\
\hline $10-01-2020$ & 9803 & 9731 & 0.73 & $10-02-2020$ & 9316 & 9654 & 3.63 \\
\hline $11-01-2020$ & 10007 & 10261 & 2.54 & $11-02-2020$ & 9164 & 9379 & 2.35 \\
\hline $12-01-2020$ & 10030 & 10123 & 0.93 & $12-02-2020$ & 9277 & 9665 & 4.18 \\
\hline $13-01-2020$ & 10172 & 10322 & 1.47 & $13-02-2020$ & 8945 & 9232 & 3.21 \\
\hline $14-01-2020$ & 9873 & 10025 & 1.54 & $14-02-2020$ & 8694 & 9085 & 4.50 \\
\hline $15-01-2020$ & 9470 & 10010 & 5.70 & $15-02-2020$ & 9183 & 9315 & 1.44 \\
\hline $16-01-2020$ & 9650 & 10106 & 4.73 & $16-02-2020$ & 9244 & 9431 & 2.02 \\
\hline $17-01-2020$ & 9830 & 10162 & 3.38 & $17-02-2020$ & 9212 & 8970 & 2.63 \\
\hline $18-01-2020$ & 10085 & 10080 & 0.05 & $18-02-2020$ & 9338 & 9175 & 1.75 \\
\hline $19-01-2020$ & 10031 & 10164 & 1.33 & $19-02-2020$ & 9505 & 9231 & 2.88 \\
\hline $20-01-2020$ & 9730 & 10203 & 4.86 & $20-02-2020$ & 9144 & 9002 & 1.55 \\
\hline $21-01-2020$ & 9582 & 9220 & 3.78 & $21-02-2020$ & 8766 & 9193 & 4.87 \\
\hline $22-01-2020$ & 9524 & 9741 & 2.28 & $22-02-2020$ & 9192 & 8955 & 2.58 \\
\hline $23-01-2020$ & 8984 & 9065 & 0.90 & $23-02-2020$ & 9260 & 10052 & 8.55 \\
\hline 24-01-2020 & 9422 & 9265 & 1.67 & 24-02-2020 & 8971 & 9210 & 2.66 \\
\hline $25-01-2020$ & 9747 & 10021 & 2.81 & $25-02-2020$ & 9022 & 9365 & 3.80 \\
\hline $26-01-2020$ & 9495 & 10042 & 5.76 & $26-02-2020$ & 9305 & 9144 & 1.73 \\
\hline $27-01-2020$ & 9662 & 9562 & 1.03 & $27-02-2020$ & 9039 & 9652 & 6.78 \\
\hline 28-01-2020 & 9570 & 9405 & 1.72 & $28-02-2020$ & 8592 & 8721 & 1.50 \\
\hline $29-01-2020$ & 9351 & 9421 & 0.75 & - & - & - & - \\
\hline $30-01-2020$ & 8908 & 9143 & 2.64 & - & - & - & - \\
\hline $31-01-2020$ & 9350 & 9412 & 0.66 & - & - & - & - \\
\hline
\end{tabular}

